\title{
RESULTS FROM X-RAY SATELLITES
}

\author{
G. Zamorani \\ Istituto di Radioastronomia \\ Via Irnerio 46, 40126 Bologna \\ Italy
}

\begin{abstract}
One of the best studied results of the X-ray observations of Active Galactic Nuclei is the statistical correlation which has been o'oserved between the X-ray and optical luminosities in these objects. In this paper I will review the present situation of the analysis of such correlation, focusing my attention on the large number of topics which are more or less directly linked to it.
\end{abstract}

\section{INTRODUCTION}

It is not easy to give today a review talk on "Results from $\mathrm{X}$-ray Satellites" for quasars and Active Galactic Nuclei (AGNs). The main reason for this is that the X-ray data for these objects (mainly from the EINSTEIN Observatory) have been extensively discussed and already presented in many Meetings in the past few years. Extensive reviews, which cover many different aspects of the X-ray properties of AGNs, such as statistical analysis of the correlations observed in various samples of quasars (radio quiet vs. radio loud), X-ray spectra, X-ray variability, $\mathrm{X}$-ray evolution and luminosity functions, have been recently published (see, for example, Zamorani 1984a, 1984b; Avni 1985a, 1985b; Mc Hardy 1985; Elvis and Lawrence 1985). As a consequence, at least until EXOSAT data are more diffusely presented and discussed in the literature, it is difficult to say something new on these subjects. This does not mean that all the possible information has already been extracted from the EINSTEIN data. On the contrary, very interesting new results are becoming available only now, as, for example, the results on the spectral data, presented at this Meeting (Elvis et al. 1986, Wilkes and Elvis 1986).

For this reason, and since other papers on a variety of various topics connected to the X-ray properties of quasars and other AGNs are presented in this Meeting, this paper will be organized as a sort of monography. Rather than showing a long list of results, not immediately related with each other, I will discuss, instead, a single observational result, the correlation between X-ray and optical luminosities, trying to show how an in depth analysis of this correlation is directly linked 
to many other both observational and interpretative data, some of which will be addressed in more detail in other papers in these Proceedings. As an example, some of the questions on which the study of the correlation between $\mathrm{X}$-ray and optical luminosities has some bearing are: a) evolution, with cosmic time, of the X-ray and optical luminosities; b) estimate of the X-ray counts and of the contribution of AGNs to the soft X-ray background;

c) variability;

d) low energy absorption in low luminosity AGNs;

e) differences in the $\mathrm{X}$-ray properties of radio quiet and radio loud quasars;

f) comparison with other existing correlations as, for example, the infrared - X-ray correlation.

In the next Sections it will be shown how our knowledge of each of these topics is connected to the study of the correlation between $\mathrm{X}$-ray and optical luminosities.

\section{THE CORRELATION BETWEEN X-RAY AND OPTICAL LUMINOSITIES}

\subsection{The Data}

Since the first EINSTEIN observations of radio quiet quasars, the existence of some kind of statistical correlation between X-ray and optical luminosities became evident (Zamorani et al. 1981). On the basis of different samples, this relationship has been described by various authors as a power $\operatorname{law}\left(\log \mathrm{L}_{\mathrm{x}}=\beta \log \mathrm{L}_{\mathrm{o}}+\right.$ constant), with $\beta$ in the range $0.6-1.0$.

The most recent and complete analysis on this topic is by Avni and Tananbaum (1986; AT) on a large sample (154 objects) of optically selected quasars. Their sample is mainly based on two complete magnitude limit samples, the $B Q S$ and the $B F$ quasars with blue magnitude limits 16.16 and 19.8, respectively. The large number of objects, and the wide dynamical range (both in luminosity and redshift) spanned by these objects allowed them to apply a very detailed statistical analysis. Figure 1 shows the $\mathrm{X}$-ray versus optical monochromatic luminosity for the objects analyzed by AT. They fit their data with a simple linear dependence of $\alpha_{\text {ox }}$ on cosmological look-back time $\tau(z)$ and on $\log \mathrm{L}_{\mathrm{O}}$ :

$$
\alpha_{\mathrm{ox}}\left(\mathrm{z}, \mathrm{L}_{\mathrm{O}}\right)=\mathrm{A}_{\mathrm{z}} \tau(\mathrm{z})+\mathrm{A}_{\mathrm{O}} \log \mathrm{L}_{\mathrm{O}}+\mathrm{A}+\text { (residual) }
$$

assuming, as initial guess, that the residuals are Gaussian distributed around the best fit line. Determining, with a parametric version of the Detections and Bounds (DB) method (Avni et al. 1980), the allowed region of the parameters $A$ and $A$, they find that $\alpha_{\text {ox }}$ depends predominantly on $L_{0}$, rather than on redshift. In particular, they find that $A_{Z}=0.0$ is fully consistent with their data. The best fijt value of the parameter $A$ corresponds to $L$ proportional to $L_{0} 0.8$. The dispersion around ${ }^{\circ}$ the best fit line (one sigma) is at least a factor of 3.0. Similar results, from a partially independent sample, have been obtained by Kriss and Canizares (1985). 


\subsection{Evolution with Cosmic Time of X-ray and Optical Luminosities}

As seen in Eq. 1, $A_{1}=0.0$ means that two objects with the same optical luminosity and different redshift statistically have the same X-ray luminosity. In other words, this implies that there is no explicit evolution with cosmic time of the ratio of X-ray to optical luminosity. On the other hand, within the framework of pure luminosity evolution models (which have become quite popular in the recent years) one can interpret the observed correlation between $\mathrm{X}$-ray and optical luminosities as the path along which single objects move, while dimming with cosmic time. In this case, a value in the slope different from 1.0 introduces an implicit dependence on redshift: while an object dims, its ratio of $\mathrm{X}$-ray to optical luminosity is changing. This is an interesting case in which the same observed correlation has two different physical interpretations as a function of the assumed evolutionary model. Theoreticians may tell us which interpretation they like more.

Moreover, as shown by Avni and Tananbaum (1982), a slope smaller than one in the correlation implies that, within pure luminosity evolution models, the cosmological evolution of the X-ray luminosity

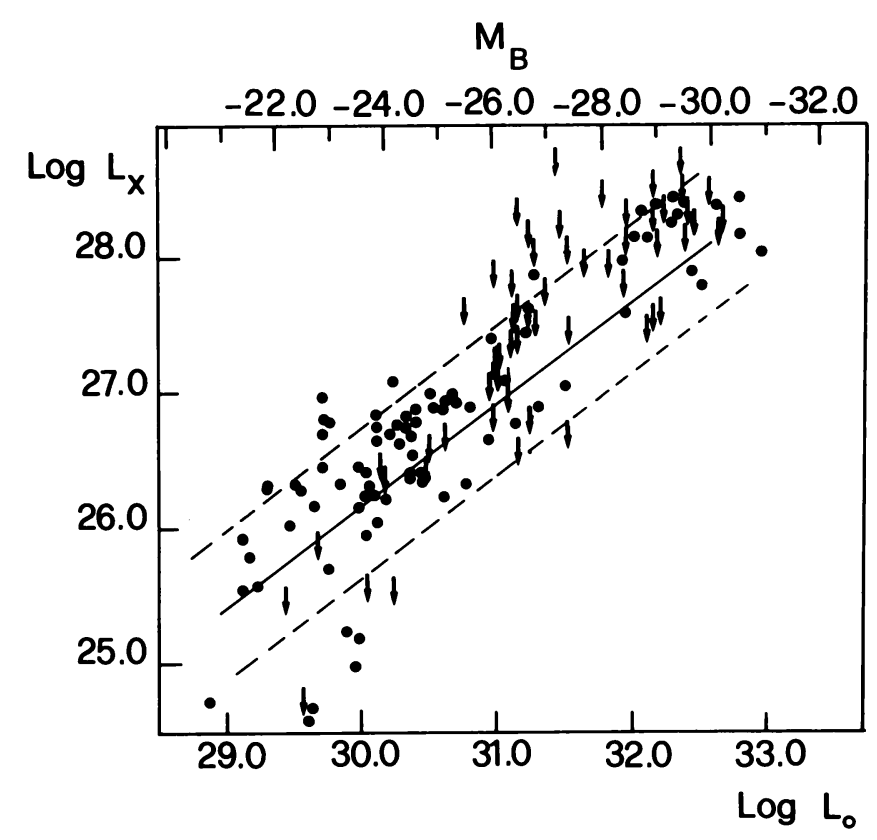

Figure 1. X-ray versus optical monochromatic luminosity for a sample of 154 optically selected quasars (see Avni and Tananbaum 1986). The solid line represents the best fit (slope 0.8). The two dashed lines represent the width (one sigma) of the Gaussian distribution of the residuals. 
function should be weaker than the evolution of the optical luminosity function. The available data seem to be in agreement with this prediction (Maccacaro, Gioia and Stocke 1984).

\subsection{Skewness of the $\alpha_{\text {ox }}$ Distribution}

In their regression analysis (see Eq. 1), AT found that the distribution of the residuals around the best fit line is not well represented by a single gaussian, but requires a significant skewness, with a longer tail at high $\alpha_{\text {ox }}$ (low $\mathrm{L}_{\mathrm{x}}$ ) and a shorter tail at low $\alpha$ (high $L_{x}$ ). This result is not axpurely $x_{\text {mathematical exercise, because }}$ the exact shape of this distribution has important numerical implications in computing, for example, the expected $X$-ray number counts. The correlation between $L_{x}$ and $L$ has been used by various authors to predict the $\mathrm{X}$-ray counts starting from the optical luminosity and evolution functions (see, for example, Avni and Tananbaum 1986; Schmidt and Green 1986). All these computations are critically affected by the detailed shape of the assumed correlation, so that the best possible analysis of the "observed" data points is crucial for this purpose. Franceschini, Gioia and Maccacaro (1985) have shown that the use of the "observed" distribution of residuals around the best fit line in the $\mathrm{L}^{-\mathrm{L}}$ plane leads to significant inconsistencies between predictions ${ }^{x}$ and observations. Most of these discrepancies can be eliminated if one assumes a narrower "intrinsic" width, which has been artificially broadened into the observed one due to a variety of external effects. The most obvious of these effects are variability and absorption (at both optical and X-ray frequencies) and errors in the estimated optical and $\mathrm{X}$-ray fluxes as well. A completely independent analysis led Zamorani (1985) to conclude that long term variability may, indeed, have produced a significant broadening in the observed distribution of the X-ray to optical fluxes in the optically selected sample. While there is no doubt that the net result is a broadening of any intrinsic correlation, a quantitative estimate of this effect can only be achieved by obtaining a better understanding of each of the causes which may determine such broadening. This is an excellent example of the necessity of linking the study of the $\mathrm{L}_{-}-\mathrm{L}$ correlation with the knowledge of other intrinsic X-ray properties of quasars.

Looking at Fig. 1, we see that while the low luminosity part of the diagram is reasonably well determined (61 Detections and 19 Bounds for $\log \mathrm{L}<31.0)$, the high luminosity region is populated by a large number of limits (34 Detections and 40 Bounds for $\log L_{0}>31.0$ ). The situation is even worse in redshift space:

\begin{tabular}{rcc}
\hline Redshift & Detections & Bounds \\
\hline $0.0-1.0$ & 71 & 16 \\
$>1.0$ & 24 & 43 \\
\hline
\end{tabular}

This implies that the shape of the distribution of residuals around the best fit line which results from the DB analysis is largely determined by low luminosity, small redshift objects. In this respect, it would be 
extremely interesting to check whether other high redshift samples (in particular the grism selected sample described in Anderson (1985) and presented at this Meeting by Margon and Anderson (1986)) are consistent with this overall description or require some minor change.

Moreover, the skewness of the $\alpha$ distribution, as determined by AT (1986), is largely required by the existence of a few detections and

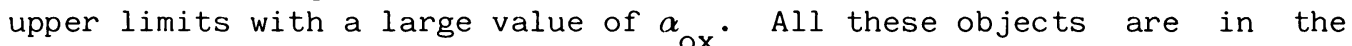
low luminosity region of Figure 1, having $\log \mathrm{L}_{<}<31.0$. Lawrence and Elvis (1982), by comparing hard and soft X-ray luminosities, suggested that a significant fraction of low luminosity AGNs might have their soft $\mathrm{X}$-ray fluxes decreased because of intrinsic absorption. No evidence for a similar effect in high luminosity objects has been found up to now. It is then possible that these high $\alpha_{\text {ox }}$ objects are simply those in which low energy absorption is becoming dominant. If this is the case, skewness might not be an intrinsic property of the $\alpha$ distribution. A possible way to test this would be to sample, with X-ray detections, the region of low X-ray, high optical luminosity. This can be achieved by obtaining deep X-ray observations of relatively bright (in optical) and high luminosity objects. It is possible that some of these observations are already available in the EINSTEIN data bank; if not, this is something that ROSAT might easily do.

\section{COMPARISON WITH OTHER STATISTICAL CORRELATIONS}

\subsection{Radio Quiet Versus Radio Loud Quasars}

It is well known (Ku, Helfand and Lucy 1980; Zamorani et al. 1981; see also Tananbaum et al. 1983) that the X-ray emission of radio loud quasars is, on average, stronger than that of radio quiet quasars. Recently, statistical associations between X-ray luminosity and extended radio luminosity (Feigelson, Isobe and Kembhavi 1984) and between X-ray luminosity and radio core emission (Kembhavi, Feigelson and Singh 1985) have been studied. A review of these results is presented by Kembhavi (1986).

The results of an extensive statistical analysis of the correlations between radio, optical and X-ray luminosities for various subsamples of radio loud quasars are presented by Worrall et al. (1986). They find a significant difference in the $\mathrm{L}_{\mathrm{x}^{-}} \mathrm{L}_{\mathrm{O}}$ correlation between radio loud with flat spectrum quasars, radio loud ${ }^{\circ}$ with steep spectrum and radio quiet quasars. This difference is such that, for a given optical luminosity, radio loud quasars with a flat radio spectrum are the strongest $\mathrm{X}$-ray emitters, while radio quiet quasars are the faintest ones. Moreover, within, each class of radio loud quasars the $\mathrm{X}$-ray luminosity (for a given optical luminosity) tends to increase with increasing radio luminosity. They also find that the fits for the steep, extended radio loud quasars improve somewhat if the radio luminosity from the core alone is used. Their conclusion is that a radio loud quasar can be thought of as a radio quiet quasar with an additional physical component producing the core radio luminosity and an "extra" X-ray emission. This extra X-ray emission would dominate the 
observed $X$-ray flux in about $80 \%$ of flat radio spectrum objects. If this is true, one might expect to see different $X$-ray spectral shapes between radio quiet and core dominated radio loud quasars (see Wilkes and Elvis 1986).

\subsection{The Correlation Between Infrared and X-ray Luminosities}

The existence of a possible correlation between the 3.5 micron nuclear flux and the X-ray emission from Seyfert 1 galaxies was first pointed out by Elvis et al. (1978), and later confirmed by Kriss, Canizares and Ricker (1980). Recently, Malkan (1984) has found that a sample of more than 50 Seyfert 1 galaxies and quasars (both radio quiet and radio loud) shows a very tight correlation between infrared and X-ray luminosities. The best fit between the two luminosities has a slope 1.0, the dispersion around the best fit (one sigma) is only a factor of 2.0 and apparently there is no obvious distinction in this correlation between radio quiet and radio loud objects. Each of these findings is at variance with the results of the analysis of the correlation between optical and X-ray luminosities (see above). The best fit corresponds to an average slope of 1.18 between infrared and $X-r a y$ frequencies. This is a confirmation of what Malkan and Sargent (1982) suggested: an extrapolation of the red/infrared power law gives an excellent prediction of the $2 \mathrm{Kev}$ flux, regardless of the ultraviolet or even the radio properties of the AGNs. (See Elvis et al. (1986) and Fabbiano et al. (1986) for more data on this subject).

These results have been taken as an indication that infrared and $\mathrm{X}$-ray emissions are produced, in all these objects, by two related (or even the same) mechanisms, probably non-thermal; at optical and UV

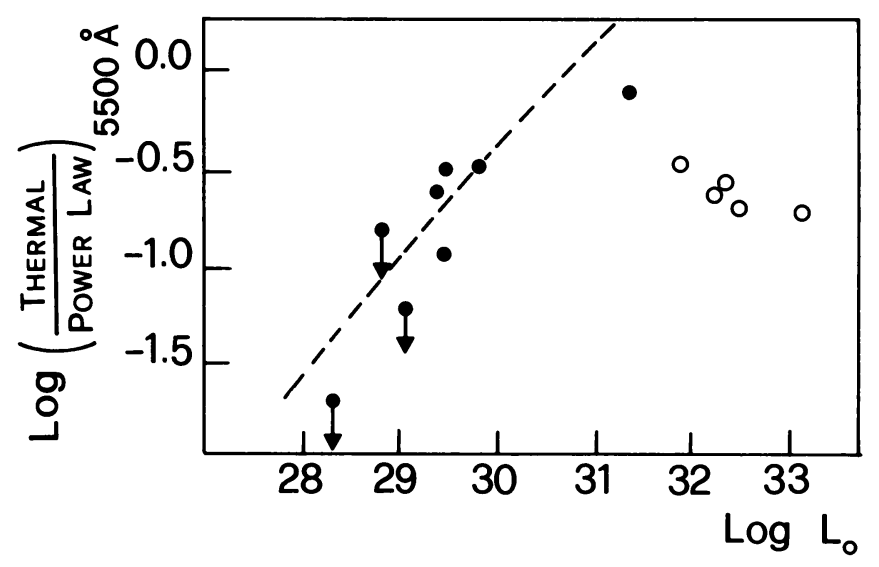

Figure 2. Ratio of thermal to power law components (at 5500 A) versus total optical luminosity. The data are taken from Malkan and Sargent 1982 (filled circles) and Malkan 1983 (open circles). The dashed line represents the trend in this diagram which would be necessary to reconcile with each other the two different slopes observed in the optical - X-ray and the infrared - X-ray correlations. 
frequencies, instead, other emission mechanisms (as, for example, Balmer continuum recombination taking place in the broad emission line regions (Richstone and Schmidt 1980; Malkan and Sargent 1982), and thermal emission from an optically thick accretion disk (Malkan 1983)) are dominant, producing the so-called blue bump and hiding the underlying, "universal" power law with a slope of the order of 1.0-1.2. Within this framework, then, the decrease of the ratio of X-ray to optical luminosities with increasing optical luminosity ( $L$ proportional to $L{ }^{2}$ ) can be understood if the ratio of the thermal to the power law component increases with increasing luminosity. This trend was, infact, suggested by the data in Malkan and Sargent (1982) (see Figure 2). Unfortunately, when five higher luminosity quasars were added to the sample (Malkan 1983), the correlation almost completely disappeared. This implies that at least one very elegant and appealing explanation for the observed difference in the $\mathrm{L}_{x}-\mathrm{L}_{0}$ and $\mathrm{L}_{x}-\overline{\mathrm{L}}_{\text {ir }}$ correlations is not so clear any more.

At this point, to clarify a little bit the situation, I think that a few obervations would be extremely important:

a) One should try to obtain the spectral decomposition (power law + thermal component) for a larger number of objects. These objects should span a range as wide as possible in luminosity. In view of the difference in the $\mathrm{X}$-ray - optical correlation for radio quiet, radio loud with flat spectrum and radio loud with steep spectrum quasars, all these classes of objects should be well represented in the sample. In costructing such a sample, objects which already have an X-ray measurement should have higher priority.

b) The infrared - X-ray correlation shown and discussed by Malkan (1984) was only for objects up to $z=0.3$. It would be extremely important to extend it to higher redshift, by obtaining more infrared observations of high redshift objects. In order not to bias the sample, not only objects which have an X-ray detection, but also objects which have only an $\mathrm{X}$-ray upper limit should be included in the sample. In this respect, it would be particularly interesting to check whether objects that appear to have an exceedingly low X-ray luminosity with respect to the optical are also amomalously infrared faint.

Before these data are available, I think we should be very careful in accepting the conclusion that infrared and $\mathrm{X}$-ray emission mechanisms are strictly related to each other.

\section{REFERENCES}

Anderson, S.F. 1985, Ph.D. Thesis, University of Washington.

Avni,Y. 1985a, Proceedings of the XII Texas Symposium on Relativistic Astrophysics, Ann. N.Y. Acad. Sc., in press.

Avni,Y. 1985b, in "Structure and Evolution of Active Galactic Nuclei", G. Giuricin, F. Mardirossian, M. Mezzetti, and M. Ramella eds., (Dordrecht: Reidel), in press.

Avni,Y., Soltan,A., Tananbaum,H., and Zamorani,G. 1980, Ap.J., 238, 800.

Avni,Y., and Tananbaum,H. 1982, Ap.J.(Letters), 262, L17. 
Avni,Y., and Tananbaum,H. 1986, Ap.J., in press.

Elvis,M., Maccacaro,T., Wilson,A.S., Ward,M.J., Penston,M.V., Fosbury,R.A.E., and Perola,G.C. 1978, M.N.R.A.S., 183, 159.

Elvis,M., and Lawrence,A. 1985, in "Astrophysics of Active Galaxies and Quasi-Stellar Objects", J.S. Miller ed., (University Science Books), p. 289.

Elvis,M., Czerny,B., Bechtold,J., and Green,R. 1986, This Volume, p.73.

Fabbiano,G., Ward,M.J., Elvis,M., Carleton,N., Willner,S.P., and Lawrence, A. 1986, This Volume, p.85.

Feigelson,E.D., Isobe, T., and Kembhavi,A. 1984, Astron.J., 89, 1464.

Franceschini,A., Gioia,I.M., and Maccacaro,T. 1985, Ap.J., in press.

Kembhavi,A. 1986, This Volume.

Kembhavi,A., Feigelson,E.D., and Singh,K.P. 1985, preprint.

Kriss,G.A., and Canizares,C.R. 1985, Ap.J., 297, 177.

Kriss,G.A., Canizares, C., and Ricker,G. 1980, Ap.J., 242, 492.

Ku,W.H.M., Helfand,D. and Lucy, L.B. 1980, Nature, 288, 323.

Lawrence, A., and Elvis,M. 1982, Ap.J., 256, 410.

Maccacaro,T., Gioia,I.M., and Stocke,J.T. 1984, Ap.J., 283, 486.

Malkan,M. 1983, Ap.J., 268, 582.

Malkan,M. 1984, in "X-ray and UV Emission from Active Galactic Nuclei", W. Brinkmann and J. Trümper eds., MPE Report 184, p. 164

Malkan,M., and Sargent,W. 1982, Ap.J., 254, 22.

Margon,B., and Anderson,S.F. 1986, This Volume, p.247.

McHardy, I. 1985, Space Sc. Rev., 40, 559.

Richstone,D.0., and Schmidt,M. 1980, Ap.J., 235, 361.

Schmidt,M., and Green,R.F. 1986, Ap.J., in press.

Tananbaum,H., Wardle,J.F.C., Zamorani,G., and Avni,Y. 1983, Ap.J., 268, 60 .

Wilkes,B., and Elvis,M. 1986, This Volume, p.261

Worrall,D.M., Giommi,P., Tananbaum,H., and Zamorani,G. 1986, This Volume , p.263

Zamorani,G. 1985, Ap.J., 299, in press.

Zamorani,G. 1984a, Proceedings of I.A.U. Symp. No. 110 on "VLBI and Compact Radio Sources", R. Fanti, K. Kellermann, and G. Setti eds., (Dordrecht: Reidel), p. 85.

Zamorani,G. 1984b, Proceedings of the International Symp. "X-Ray Astronomy '84", M. Oda, and R. Giacconi eds., (Institute of Space and Astronautical Science - Tokyo), p. 419.

Zamorani,G., Henry,J.P., Maccacaro,T., Tananbaum,H., Soltan,A., Avni,Y., Liebert,J., Stocke,J., Strittmatter,P.A., Weymann,R.J., Smith,M.G., and Condon, J.J. 1981, Ap.J., 245, 357. 
Malkan : To update what you said about the correlation of the thermal/ nonthermal ratio with luminosity presented in Malkan \& Sargent : though it is poor for quasars, our new analyses of Seyfert 1's strengthen the correlation at low luminosities. That is, high and low-luminosity quasars appear to have blackbody/power-law ratios (at $5450 \mathrm{~A}$ ) of 0.5 to 1 , whereas many Seyfert 1 's have ratios of 0.1 . So I think it's still possible this correlation may explain the $\alpha_{o x} / L$ trend, and the Baldwin effect.

Wampler : There are a few (2) broad absorption line (BAL) quasars known that have M II $\lambda 2800$ in absorption. The M II $\lambda 2800$ absorption line has a central depth of only about $50 \%$ and seems to separate the emission of a small central source from that of a larger source. At least in the case of PG $1700+518$ the underlying radiation from the small source is a power law $\left(f_{\nu} \propto v^{-\alpha}\right.$ ) with $\alpha=1.2$. Other BAL quasars may be useful in separating the various components that contribute to the optical continuum and aid in understanding of the general connection between the IR-opt-X ray portions of the continuum.

Segal : An apparently high correlation between the luminosities in two different frequency bands can easily be the spurious consequence of a not necessarily valid cosmology in which flux varies rapidly with redshift since the derived luminosities will then have this flux-redshift function as a common factor. Can you exclude this possibility in the cases you report.

Zamorani : Yes, I think I can - infact, for the data that I have shown there is a highly significant correlation also between the observed fluxes, which are obviously independent of any assumption on cosmology. 